\title{
Recherche clinique dans les hôpitaux non universitaires
}

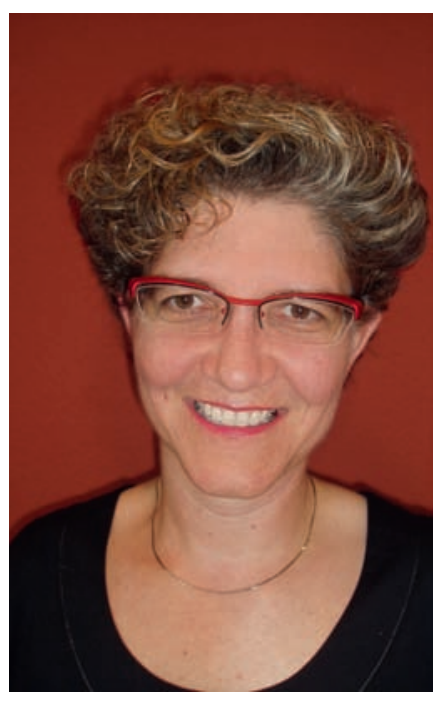

Le déchiffrage du génome humain fournit une base totalement nouvelle à la compréhension des maladies. Mais pour améliorer concrètement le traitement des patients, la recherche fondamentale et les connaissances théoriques du génome ne suffisent pas. C'est pourquoi ces nouvelles connaissances n'ont pas encore porté leurs fruits, à de rares exceptions près, malgré les grands espoirs qui les ont précédées. Sont très vite apparus des goulets d'étranglement («bottlenecks») qui retardent l'application des connaissances de médecine moléculaire dans des projets thérapeutiques validés. Un de ces obstacles est l'absence d'une culture de recherche clinique solidement étayée.

En ce moment, dans le monde entier, des institutions publiques chargées de la recherche soutiennent des programmes de développement et de revitalisation de la recherche clinique. Ainsi le $7^{\text {e }}$ programme cadre pour la recherche et la technologie de l'Union européenne - l'instrument principal de promotion de la recherche en Europe - dédie toute une thématique à la santé [1]. Dans l'introduction du texte d'annonce on peut lire: «Le but principal du thème ssantés est l'amélioration de la santé des citoyens de l'Union européenne. [...] Ce but doit être atteint grâce à une recherche de translation, c'est-à-dire à une transposition des résultats de la recherche fondamentale dans les applications cliniques.»

Pour stimuler la recherche clinique en Suisse, le Fonds national a, dès 2007, établi un programme pour mettre en place des unités de recherche cliniques (Clinical Trial Units, CTU)
[2]. De telles unités existent dorénavant dans tous les hôpitaux universitaires suisses ainsi qu'à Saint-Gall. Elles mettent à disposition des chercheurs tout un faisceau de connaissances spécialisées telles qu'épidémiologie, statistique, pharmacologie. Elles établissent les contacts nécessaires avec les autorités et les commissions d'éthique, afin de faciliter le déroulement des études cliniques. Grâce à une interconnexion efficace des unités dans le cadre de la «Swiss Clinical Trial Organisation», on peut espérer des effets de synergie entre elles. Ce ne sont pas seulement les CTU, mais également les hôpitaux non universitaires de Suisse qui doivent être intégrés dans ce réseau d'études cliniques.

Innover en médecine n'est possible que grâce à la participation active à la recherche clinique. Participer activement signifie aussi bien effectuer une étude pour le compte d'autrui qu'avoir la possibilité de réaliser ses propres idées! Durant ces prochaines années, des innovations verront le jour non seulement en médecine de pointe mais aussi dans le domaine des soins de base. Il est essentiel que les hôpitaux suisses ne se contentent pas seulement d'appliquer ces innovations, mais contribuent à les développer, dans l'intérêt de leurs patients.

Barbara Biedermann*

\footnotetext{
Références

1 http://cordis.europa.eu/fp7/home_en.html.

2 www.snf.ch/E/funding/projects/ctu/Pages/ default.aspx.
}

* La doctoresse Barbara Biedermann, p.-d., est médecin adjointe en médecine moléculaire à l'Hôpital cantonal de Bruderholz BL. Elle défend les intérêts des hôpitaux non universitaires dans le cadre de la création d'une «Swiss Clinical Trial Organisation».
«La recherche clinique dans un hôpital non universitaire», tel est le thème d'un atelier d'une journée qui aura lieu le 16 octobre 2008 au Bruderholzspital BL. L'atelier veut montrer quelles sont les possibilités réalistes et praticables d'exercer une activité de recherche indépendante et fructueuse, même pour les hôpitaux non universitaires. Un meeting consacré à la stratégie permettra aux participants de fixer les buts, à moyen et long terme, d'un réseau de recherche suisse, dans le domaine des soins de base. Renseignements: PD Dr. med. Barbara C. Biedermann, Medizinische Universitätsklinik, Kantonsspital Bruderholz, 4101 Bruderholz, tél. 06143631 44, e-mail: barbara.biedermann@unibas.ch. 